\title{
Vašek Chvátal: A Very Short Introduction
}

\author{
David Avis ${ }^{1}$, Adrian Bondy ${ }^{2}$, William Cook ${ }^{3}$, and Bruce Reed ${ }^{1,4}$ \\ ${ }^{1}$ Computer Science, McGill University, 3480 University, Montreal, H3A 2A7, QC, Canada. \\ e-mail: avis@cs.mcgill.ca \\ ${ }^{2}$ Université Claude-Bernard Lyon 1, Domaine de Gerland, 50 avenue Tony Garnier, 69366 \\ Lyon Cedex 07, France \\ ${ }^{3}$ Industrial and Systems Engineering, Georgia Tech, 765 Ferst Drive, Atlanta, GA, 30332, \\ USA \\ ${ }^{4}$ Project Mascotte, INRIA + CNRS (Lab I3S), Sophia Antipolis, France
}

Abstract. This is the story of a man named Vašek.

Key words. Vašek, Chvátal

\section{Young Genius-Notes by AB}

Raised in Plzeň, renowned for its brewery, Vašek studied mathematics at Charles University in Prague under the tutelage of Zdeněk Hedrlín, along with Pavol Hell, Luděk Kučera and Jarik Nešetřil. There he was trained in the algebraic aspects of graph theory, publishing his first paper [14], appropriately on rigid digraphs (those with only trivial endomorphisms), at the tender age of 19 (Fig. 1).

In August 1968, Vašek and his then wife Jarmila decided that their future lay elsewhere but in the clutches of his homeland's unwelcome guests. After a brief stop in Vienna and an encounter with a ghost in Hyde Park, Vašek made landfall in Fredericton, New Brunswick, where he set his mind to positional games on hypergraphs and Ramsey-type problems. It was at the pivotal Calgary meeting the following summer that many of my generation first met one another, and this was so for Vašek and myself. Vašek was already a seasoned researcher, having written some half-dozen papers on a variety of topics. The following autumn, he enrolled as a Ph.D. student with Crispin Nash-Williams. It took him just a year to obtain his doctorate. He sailed through the notorious comprehensive exams that Crispin was in the habit of setting, and found time between bouts of research to drink beer (Fig. 2).

Vašek's doctoral thesis, on Hypergraphs and Ramseyian Theorems, gave rise to several publications, notably [16]. Vašek subsequently wrote a series of papers with Frank Harary where they introduced and studied generalized Ramsey numbers [39-41]. 
D. Avis et al.

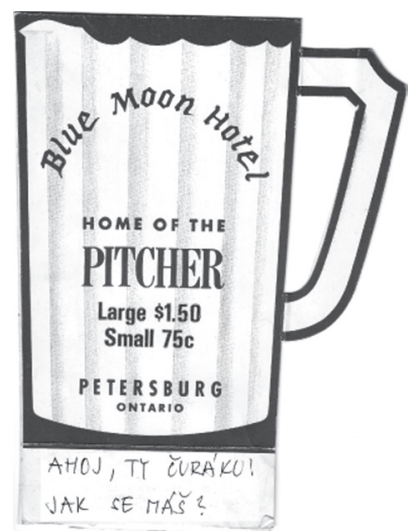

During that year, two mathematicians were to have a significant influence on the direction of Vašek's future research. One was Nash-Williams, the other Jack Edmonds. Nash-Williams gave a graduate course on Hamilton cycles. As a support for the course, he had prepared a 58-page manual of definitions and notation, including formal definitions of ordered and unordered pairs, the distinction between circuits (sequences) and circuitoids (subgraphs), as well as idiosyncratic notation, the most memorable of which was the "lampshade" of a set. Only the most complicated of Greek letters, $\xi, \eta$ and $\zeta$, would do for names of vertices. Despite or perhaps because of this, the course left its mark on all of us. Vašek inherited Nash-Williams' insistence on precision, but preferred the brisk clear style, expressive language, and crisp notation that exemplify his writings and lectures. (In explaining Zykov's proof of Turán's theorem to me many years ago, he referred to a certain stable set as

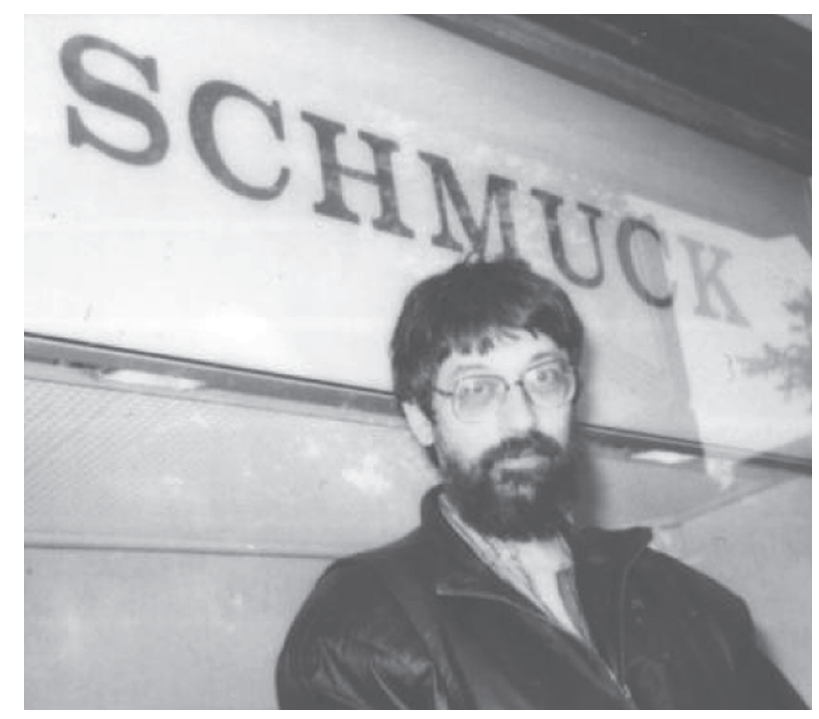

Fig. 1. A short introduction 


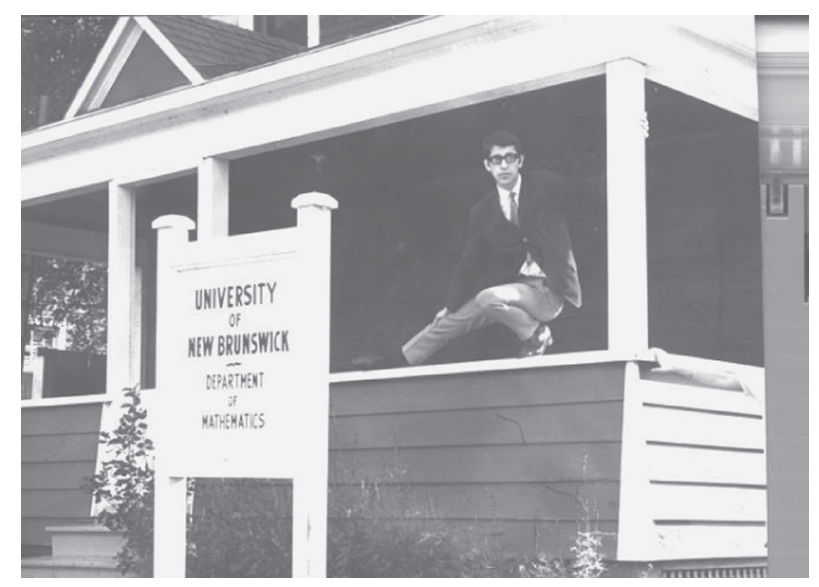

Fig. 2. Fall 1968

"fly-shit" - that proof is now engraved in my memory. More recently, sharks and swimmers were invoked during a talk on instances of the travelling salesman problem. His pedagogical talents extend to everyday life. Once, having been served a very tough steak, he carefully shaped it into the form of a shoe sole and left it lying eloquently on the plate.) One of the theorems covered by Nash-Williams was Pósa's sufficient condition for hamiltonicity, a weakening of Dirac's degree condition. Shortly afterwards, Vašek came up with a best possible result of this type [17]. (It was in searching for an algorithmic proof of this theorem a few years later that we conceived the Hamiltonian closure operation [11].) Vašek wrote several influential papers on the topic of Hamilton cycles, notably [22], where he introduced the notion of toughness, a graph being $t$-tough if no more than $k$ components result from the deletion of fewer than $(k+1) t$ vertices. There he proposed the conjecture that every $t$-tough graph is Hamiltonian provided that $t$ is large enough. Even though his initial guess of $t>3 / 2$, and later $t=2$, proved to be too optimistic [7], the conjecture remains very much alive. Vašek reduced his Erdős number to one by coming up with an elegant sufficient condition for Hamiltonicity in terms of the stability number and connectivity [38], and this during a car ride with the P.G.O.M. from Pullman to Spokane, WA (Fig. 3).

With characteristic courtesy, Vašek thanks Louise Guy for her steady driving. At a Summer Research Institute in Quebec City in the summer of 1971, Vašek constructed infinite families of hypoHamiltonian graphs [21], but also found time to learn French, fall in love with his French teacher, and write a prize-winning short story, Déjà Vu.

For the record, it should be mentioned here that he had a hand or two in an $\mathcal{N} \mathcal{P}$-completeness result on Hamilton cycles [77], namely that deciding whether a graph has a Hamiltonian square is $\mathcal{N} \mathcal{P}$-complete, published by one P. (for Polly) Underground whose main profession was not mathematics. (Polly also makes an appearance in Vašek's highly regarded and much used text Linear Programming.) There was also an unpublished note entitled "A sufficient condition for a graph to be 


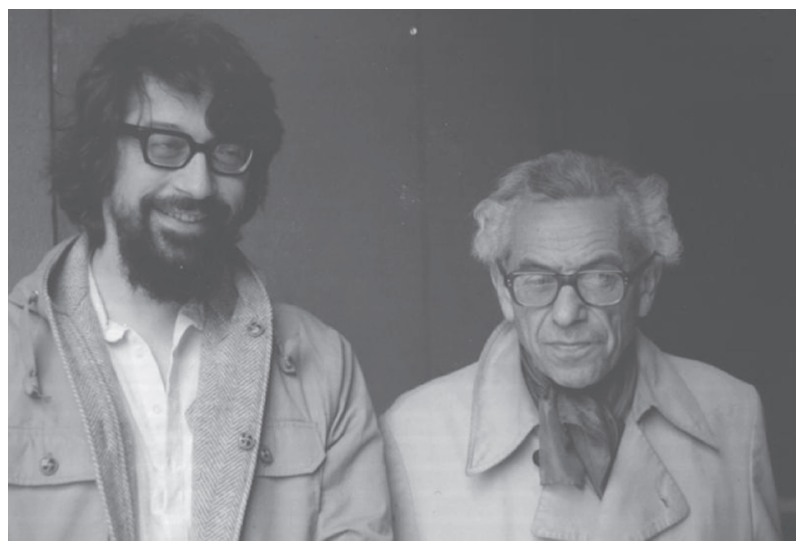

Fig. 3. With a co-author

almost complete", which showed that a certain sufficient condition for hamiltonicity, touted by its author at a Florida meeting as superior to existing conditions (such as Vašek's degree condition) because high density was not required, was satisfied only by circuits or extremely dense graphs.

It was Edmonds who introduced Vašek to the world of linear programming. Vašek quickly realized its potential role in $\mathcal{N} \mathcal{P}$-hard problems such as the Hamiltonian and travelling salesman problem [19, 20]. Indeed, as David, Bruce and Bill recount below, the connections between linear programming and perfect graphs and Hamilton cycles have occupied Vašek's fertile mind for the past 35 years. Among his early results was the one-two-three theorem. Defining a graph to be weakly Hamiltonian if there is a function on its edge set satisfying four sets of linear inequalities that are valid for any Hamiltonian graph (three of these are straightforward, while the fourth introduced the important notion of comb inequalities), he proved that a weakly Hamiltonian graph is necessarily 1 -tough, has a 2 -factor, and is 3-cyclable (any three vertices lie on a cycle).

As Vašek explains in a tribute to Claude Berge [33], he first encountered the "maître" in a Plzeň bookshop in 1964, where a Russian translation of Berge's first book, lying in wait for him, seduced him into graph theory. He met Berge in person at the Calgary meeting five years later, and they became close friends (Figs. 4, 5).

At the Hypergraph Seminar in Columbus in 1972, organized by Berge and Dijen Ray-Chaudhuri, he proposed a now-famous and still unsolved conjecture on hereditary hypergraphs [23]. It is informative to quote the Math. Review by Paul Erdős, no stranger to hypergraph extremal problems:

The author states the following surprising conjecture: Let $F$ be a family of subsets of a finite set $S$ such that if $X \in F, Y \subset X$ then $Y \in F$; consider a subfamily of $F$ any two sets of which intersect and which has the largest number of sets; we can obtain such a family by considering all $X \in F$ which contain a suitable element t of $S$. The author proves several special cases of this beautiful conjecture. 


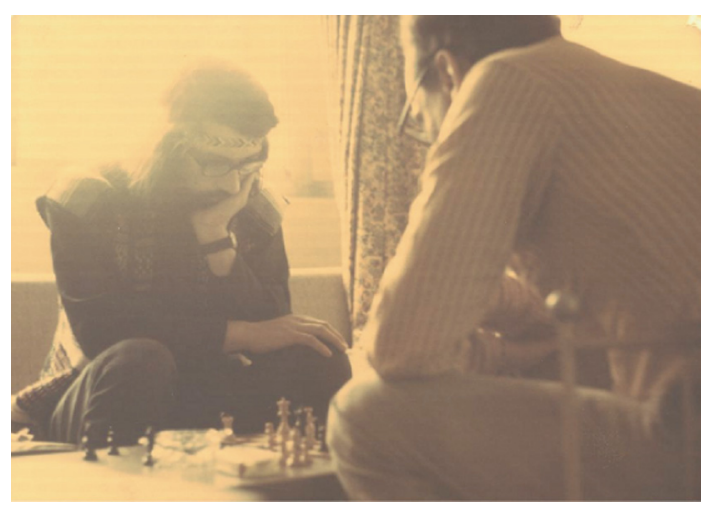

Fig. 4. With Claude Berge

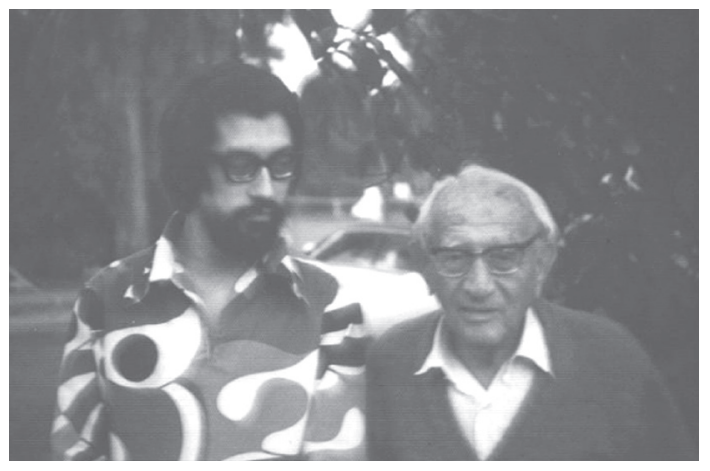

Fig. 5. With George Polya

In a typically extravagant gesture, Vašek [42] offered $\$ 10.00$ for a proof or counterexample. (His coauthors of this collection of combinatorial problems, David Klarner and Donald Knuth, wisely appended the disclaimer "All cash awards are Chvátal's responsibility". The reward offered for settling his conjecture on toughness was somewhat more generous.) The hereditary hypergraph conjecture has been the topic of some twenty articles (see http://www.users.encs.concordia.ca/ chvatal/conjecture.html).

Also at the hypergraph seminar, having failed in our attempt to "get the young genius drunk" (and then proceed to turn him into an alcoholic, thereby eliminating him from mathematical competition), he joined forces with Laci Lovász, proving the pretty result that every digraph has a semi-kernel, a stable set reachable from every vertex in at most two steps [43].

It is hard to do justice to Vašek's prolific and remarkably eclectic output during the late 1960s and early 1970s. To mention just three further examples, he found a colourful attractive extension of the Gallai-Roy theorem on paths in digraphs [18], proved a theorem in plane geometry [25] - commonly referred to as Chvátal's Art Gallery Theorem - which determines the number of guards required to survey the 


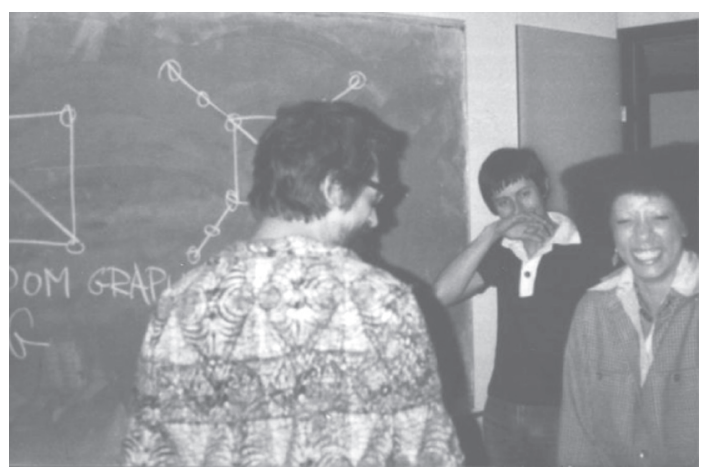

Fig. 6. With a visiting speaker

walls of a polygonal art gallery (and has prompted much research), and constructed the smallest triangle-free 4-chromatic 4-regular graph, a beautiful graph now known as the Chvátal graph [15] (Fig. 6):

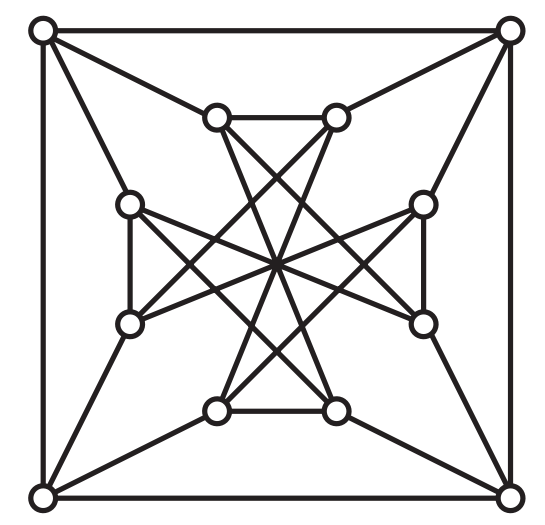

The promise of those early years has been amply fulfilled.

\section{Linear Programmer-Notes by DA}

In a typically succinct fashion, the abstract of Vašek's 1984 technical report "Cuttingplane proofs and the stability number of a graph" [31] states his position on, and contribution to, the connection between linear programming and combinatorics.

Many claims in combinatorics can be stated by saying that every integer solution of a specified system of linear inequalities satisfies another specific inequality. Such claims can be proved in a certain canonical way involving the notion of cutting planes. We investigate the structure of these proofs in the particular case 
where the claim is that a specified graph contains at most a specified number of pairwise nonadjacent vertices.

Two of the paper's important results will be stated below, but first let us look at their origins. Vašek became interested in linear programming during his postdoctoral year, 1970-1971. Its usefulness as a tool for combinatorial optimization had been spectacularly demonstrated by Edmonds a few years earlier in his efficient solution of the weighted matching problem for graphs [51]. Of particular interest was Edmonds' use of the duality theorem of linear programming to produce "good characterizations", or what we would now call "certificates of optimality". In the brief period before the dark clouds of NP-hardness appeared, there was considerable optimism that this method would soon knock off a number of other apparently similar problems. Three such graph theory problems that were of particular interest to Vašek were finding a largest stable set, a minimum vertex colouring, and a Hamiltonian circuit.

Vašek spent the summer of 1971 in Quebec City, the fall at McGill, and the winter of 1972 at Stanford. Under the slogan "combinatorics = number theory + linear programming," Vašek set out a general framework for this approach in "Edmonds Polytopes and a Hierarchy of Combinatorial Problems" [19]. He defined the closure of a system of linear inequalities to be the set of inequalities valid for its integer solutions. He proved that all such inequalities could be obtained by a finite process of taking positive combinations of inequalities and rounding. This procedure gave rise to a natural notion of the rank, now known as Chvátal rank, of the inequality, and indeed for an integer program itself. Although the main theorem can be proved using the finiteness of Gomory's integer programming algorithm, [54], Vašek gave an elementary proof that is simple enough that it has found its way into several integer programming textbooks. The integer inequalities generated are now known as "Chvátal-Gomory cuts". And the paper contains much more, including, naturally, applications to independent sets, graph colouring, and Hamiltonian circuits. The paper was submitted in May 1972.

During the time the paper was written, Stephen Cook [47] and Richard Karp [64] announced results that were to change the landscape forever. These results are mentioned in the revised version of the paper, submitted in May 1973, in the concluding remarks. After citing them he writes:

One may be tempted to believe that each class of zero-one linear programming problems having a bounded rank possesses a polynomial time algorithm. If this were true then, in particular, there would be a polynomial time algorithm searching for the largest independent sets in perfect graphs.

For in the summer of 1972, Vašek had attended a meeting in Columbus, Ohio where Lovász [69] presented his proof of the weak perfect graph theorem. As Adrian has mentioned, Vašek had heard of perfect graphs from Berge several years earlier. Lovász's result (and independent work of Fulkerson) connected them to linear programming. This connection was spelled out in the subsequent paper "On certain polytopes associated with graphs" [24], and was fortuitous. NP-hardness had essentially eliminated the possibility of extending Edmonds' methods to obtaining polynomial time solutions to Vašek's favourite problems for general graphs. The 
game now was to find large classes of interesting graphs for which hard problems were tractable. Perfect graphs were known to include comparability graphs, chordal graphs and line graphs of bipartite graphs, for which finding a maximum independent set or a minimum vertex colouring were solvable in polynomial time. A perfect class of graphs to study was at hand. In the winter of 1972 Vašek started to consider the problem of recognizing perfect graphs in polynomial time. He noticed that the first two of these special classes of perfect graphs could be recognized by placing the vertices in a special ordering. But I digress - perfect graphs will be covered by Bruce in the next section. The paper [24] had a number of other interesting results, including this gem: two stable sets in a graph are adjacent in the corresponding stable set polytope if and only if their symmetric difference defines a connected graph. As a corollary, by considering line graphs, a similar statement can be made for matchings. Vašek's contributions to linear programming continue with his arrival at Stanford in the fall of 1974.

The squeal of tires, a white Ford Mustang, and the sound of the Beatles "Rubber Soul" announce Vašek's arrival at Encina Hall, the bucolic location of Stanford's Department of Operations Research. The department was home to George Dantzig himself, the father of linear programming, and benefactor of most of the doctoral students in the department, myself included. I was then working on a dissertation in queuing theory. Suddenly Vašek appeared in my office, cigarette holder in hand, and sporting a red headband. He asked me to proof-read "Some linear programming aspects of combinatorics" [26]. It had an abstract that read, rather unpromisingly:

This is the text of a lecture given at the Conference on Algebraic Aspects of Combinatorics at the University of Toronto in January 1975. The lecture was expository, aimed at an audience with no previous knowledge of linear programming.

I was less than enthusiastic. To put this in context, like most of my classmates, I thought I had seen the last of linear programming with the five hour comprehensive exam the year before. His paper opened my eyes. What we had considered to be a dead subject was here being used to prove Sperner's lemma, the ErdösKo-Rado theorem, and ... the pigeon hole principle!? It also contained a digest of his two papers mentioned above. The message was clear: linear programming belonged in the tool kit of every discrete mathematician. I was hooked - and Vašek had a doctoral student (Fig. 7).

Stanford was famous for devouring assistant professors: 3 years of teaching the courses no one else wanted, then off to a new job. Vašek had the unenviable task of teaching linear programming to Master's students in operations research, for most of whom mathematics was not their strong point. Descriptions of the subject at the time tended to be rather dry, technical, unintuitive and use lots of unfriendly notation. What was needed was a simple, intuitive but precise introduction to the subject that would be useful to student and working mathematician alike. During the Christmas vacation of 1975, that was precisely what Vašek wrote. I had spent the vacation in Mexico with some friends, and when I returned Vašek told me he had written a book on linear programming. I was sure he was joking, but he gave me his manuscript, which was a little over 100 pages long. I started reading it that evening, and kept reading until it was finished. It was a masterpiece of technical 


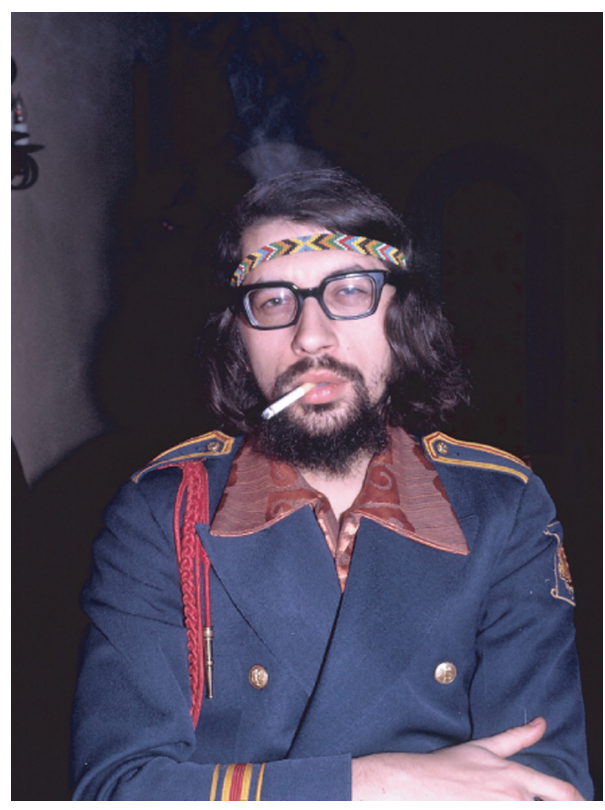

Fig. 7. Sergeant Chvátal, taken by Adrian

writing. The manuscript circulated quickly. Everybody read it, everybody loved it, and everybody said, "It's perfect, but, if you would just add a chapter on ... it would be even better." Meanwhile, we students speculated that such an upstart would never get tenure by showing up the master ${ }^{1}$.

Vašek wanted some new results that his book would be the first to contain, but the subject had remained relatively dormant for some time. Then in his 1976 doctoral thesis, Bland announced pivot rules for the simplex method that did not cycle. A particularly simple rule involved merely choosing each candidate variable with smallest subscript. In the summer of 1976 Vašek returned to Montreal to work at the Centre de Recherches Mathématiques. Having just finished the first draft of my thesis, and I expected it would be a month of Sundays before he got around to reading it. Suddenly Vašek called me up and invited me to come to Montreal. We would work together to see whether Klee and Minty's examples also applied to Bland's rule, I would repeat the Kuhn-Quandt experiments for it, and he would read my thesis. It was half a lifetime ago: Vašek was 30. Ivo Rosenberg was away and kindly let us stay in his house. To give Vašek more reading time, I was his driver in a clapped out car that barely made it up the hill to the CRM. Filling the tank was a lengthy affair as it also required filling the oil and changing the spark plugs. The blur of cafés, restaurants, strip clubs, beautiful women and $24 \mathrm{~h}$ flower shops quickly convinced me that Montreal was where I would look for a job. We ended up getting the results on Bland's rule, but needless to say the paper [6] did not get written until after we went back to California.

\footnotetext{
1 We were wrong, he got it.
} 


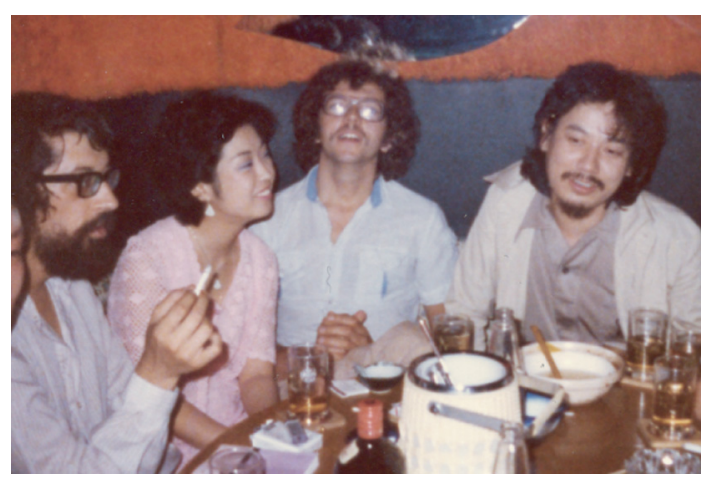

Fig. 8. With Jin, David and a bijin

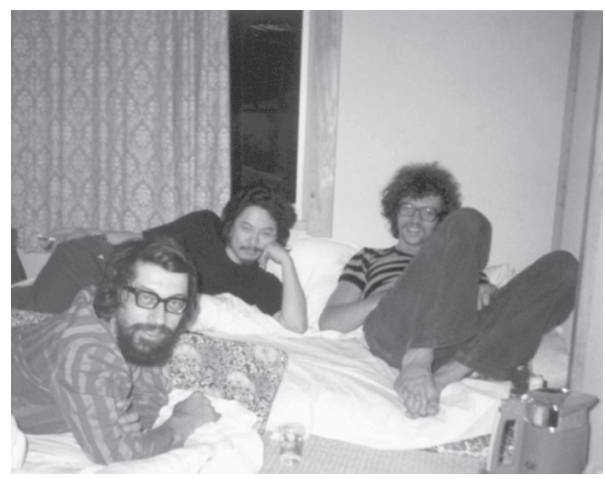

Fig. 9. Nikko

Returning to Stanford in the Fall of 1976, Vašek diligently set out to keep all of those people happy who had read his manuscript. He first learned, then wrote chapters on, implementation, networks, games, applications, geometry, generalized upper bounding, Dantzig-Wolfe decomposition, branch and bound and so on. The work continued after he moved back to Montreal in 1977, first at the Université de Montréal, then at McGill from 1978. The long, brutal Montreal winters of those days provided plenty of time to work on the book, but the summers were another matter.

In the summer of 1978 Vašek summoned me to Paris from Belgium, where I was doing a postdoc. We discussed the possibility of making our living off the glitterati of Saint Tropez during an exquisite lunch with Jean-François Maurras, that lasted most of an afternoon. The next day Vašek and I jetted off to the Côte D'Azur to try our luck with a new career. Needless to say, we were back at McGill by Autumn. The following summer we went to Japan and met Jin, as described in the Preface to this issue (Figs. 8, 9).

During the next few years, Vasek dutifully devoured all of the material suggested to him for his expanded book, thoroughly digested it, and then rewrote it in the same clear concise style that characterized his original manuscript. History, 
ever willing to repeat itself, records that while the text was in its final stages, Khachian [65] announced the first polynomial time linear programming algorithm, the ellipsoid method. The ellipsoid algorithm made it into the text as an appendix, an exposition that is still one of the best available, and is widely reproduced. When Linear Programming [30] finally appeared in 1983, it was immediately proclaimed the definitive text on the subject. The original manuscript, recognizable, but not quite intact, is the first part of the book and a complete course in itself. On the cover was a reproduction of a painting by his close friend, the artist François de Lucy, which was an image that Vašek said reminded him of all the paper crumpled up along the way.

For the next thread of the story, we go back to Stanford. In the mid-1970s there was still hope that the $P=N P$ question might be tractable. The conventional wisdom was that these classes were different, and computer scientists were using the tools of their trade to try and resolve the issue. If $P$ and $N P$ really were different, then cutting planes ought to be weak for solving hard integer programs. This is what Vašek proved for a related, but weaker proof system, for one of his favourite problems, determining the size of the largest independent set in a graph [19]. The paper is long and the proof is difficult. The paper contains a prescription for generating random graphs for which with high probability every proof in this system has exponential length. Similar results, for which the arguments are much simpler, were given for the knapsack problem in [29]. The much-cited paper on randomly generated hard examples for resolution [46], written jointly with Szemerédi, is the climax of this series.

Now we return to Vašek's 1984 technical report, the abstract of which began this section, and its two main results. For any vertex $v$ of a graph $G$ associate a variable $x_{v}$, and consider the systems

$$
\begin{aligned}
& \sum_{v \in C} x_{v} \leq 1 \text { for all cliques } \mathrm{C} \text { in } \mathrm{G} \\
& -x_{v} \leq 0 \text { for all vertices } \mathrm{v} \text { of } \mathrm{G}
\end{aligned}
$$

and

$$
\sum_{v \in G} x_{v} \leq \alpha(G)
$$

where $\alpha(G)$ is the size of the largest independent set in $G$. His first theorem reads:

Theorem 1. There are arbitrarily large graphs $G$ and a positive constant $\epsilon$ such that the depth of every cutting-plane proof of (2) from (1) exceeds $\epsilon$.

His second theorem reads:

Theorem 2. There are arbitrarily large graphs $G$ with $\alpha(G)=2$ such that the depth of every cutting-plane proof of (2) from (1) exceeds $\frac{1}{3} \ln n$. 


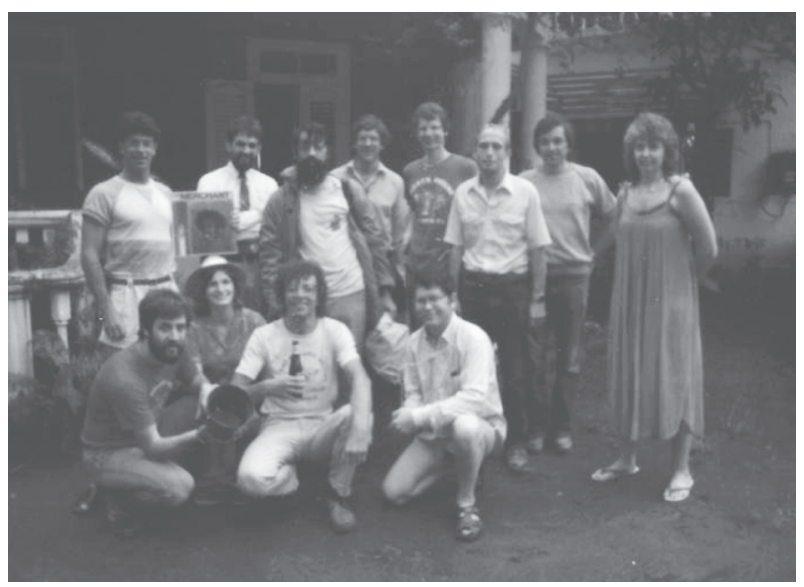

Fig. 10. The first Barbados ever

The method of the 1984 paper was extended and applied to a wide variety of other combinatorial problems in a subsequent paper with Bill Cook and Mark Hartmann [37]. One of the applications was to two objects dear to my heart, the cut and metric polytopes, so I cannot resist including it here. The cut polytope is the convex hull of zero-one vectors defined by the edge sets of cuts in a complete graph. The metric polytope its natural relaxation defined by considering all triangle inequalities on the same set of variables. An important class of facets of the cut polytope (see for example [50]), called pure hypermetric facets by Michel Deza, are defined for any complete graph $H$ on $2 t+1$ vertices by

$$
\sum_{e \in E(H)} x_{e} \leq t(t+1)
$$

The case $t=1$ is an example of a triangle inequality. It is shown in [37] that the depth of any cutting place proof of (3) from the metric polytope is at least $(t-1) / 2$.

By 1983 with the book out of the way, Vašek started to focus his attention on perfect graphs, and the strong perfect graph conjecture. He gathered an exceptional group of talented graduate students, the exploits of whom Bruce describes below. For me, a sabbatical year in Japan in 1983-1984 put me so far behind the group that to catch up was impossible. Soon after Vašek was to leave Montreal, and it would be almost 20 years before he returned:

\section{Un Canadien errant}

Banni de ses foyers

Parcourait en pleurant

Des pays étrangers ${ }^{2}$.

It was the end of an era (Fig. 10).

${ }^{2}$ From "Un Canadien Errant", by Antoine Gérin-Lajoie (1837). 


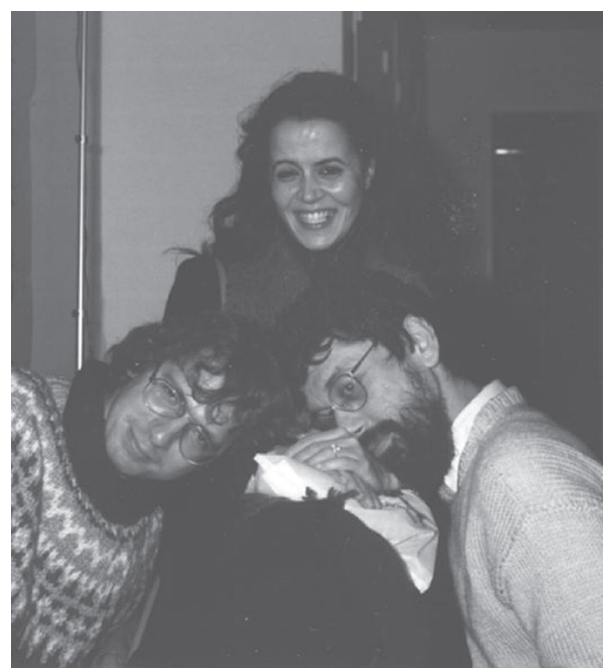

Fig. 11. With Bill and Najiba

\section{Perfectionist-Notes by BR}

In typically irreverent fashion, Vašek's book Linear Programming begins with a quote which captures one of the strengths of this polished gem.

Suppose you want to teach the cat concept to a very young child. Do you explain that a cat is a relatively small, primarily carnivorous mammal with retractile claws, a distinct sonic output, etc.? I'll bet not. You probably show the kid a lot of different cats saying kitty each time until it gets the idea. To put it more generally, generalizations are best made by abstraction from experience ${ }^{3}$.

That he routinely introduces complex ideas via a sequence of simple and crystalclear examples is just one of the reasons Vašek is a great teacher. Once he had settled at McGill, Sensei gathered around him a group of disciples, in whom he instilled his passion for perfect graphs. We were attracted to him like moths to a flame and began to burn with the same inner fire (Fig. 11).

Perfect graphs were defined by Berge, a very close friend whom Vašek sometimes called Master. Berge's definition was motivated by a question posed by Shannon on the asymptotic transmission rates of noisy channels [75]. A graph $G$ is said to be perfect if for each of its induced subgraphs $H$, there is a set of $\alpha(G)$ cliques covering its vertices. In 1960, Berge observed that a chordless cycle of length $2 k+1, k \geq 2$, cannot be covered by $k$ edges. He also observed that the complement of such a cycle cannot be covered by two cliques. Hence any graph containing an induced odd cycle of length at least five or the complement of such a cycle is not perfect. After searching in vain for other examples of imperfect graphs, Berge made the following conjecture [8]:

3 R.P. Boas, Am. Math. Mon., 88, 727-731 (1981). 


\section{A graph $G$ is perfect precisely if neither $G$ nor $\bar{G}$ contains an induced odd cycle} of length at least five.

This conjecture became known as the strong perfect graph conjecture. The term weak perfect graph conjecture was reserved for its corollary

If a graph is perfect then so is its complement.

which was proved by Lovász in 1971 [68].

Lovász's result is equivalent to the statement that the vertices of every induced subgraph $H$ of a perfect graph can be covered by $\alpha(H)$ cliques. Hence the depth of the cutting plane proof of (2) from (1) is zero for all such graphs. This is equivalent to saying that for any $0-1$ weight vector $w$, we can choose an integer-valued $x^{*}$ in the fractional stable set polytope of $G$ [the polytope defined by the set (1) of inequalities] which maximizes $w x$ over this polytope.

In [24], Vašek noted Lovász's proof of the WPGC implies that much more is true. He showed that we can actually choose an integer valued $x^{*}$ maximizing $w x$ for any weight vector $w^{4}$. Put another way, the fractional stable set polytope has only integer vertices. Thus, we can characterize some of those $0-1$ matrices $A$ such that $A x \leq 1, x \geq 0$, has only integer vertices as the fractional stable set polytopes of perfect graphs. It turns out that all such matrices can be characterized in this fashion (provided we remove redundant constraints). Thus, characterizing perfect graphs yields a characterization of a natural class of integer programs which are solvable in polynomial time using Khachian's linear-time algorithm for linear programming. This consequence of Vašek's observation spurred interest in perfect graphs in the combinatorial optimization community.

Despite the strength and beauty of Vašek's result, it does not imply that we can determine $\alpha(G)$ for perfect $G$ by constructing and solving this LP, as the number of cliques in a perfect graph $G$ with $n$ vertices may be as large as $2^{n / 2}$. However, in 1979 Grötschel et al. [58] wrote a seminal paper which showed that actually the result can be combined with the ellipsoid method to find maximum weight stable sets in perfect graphs in polynomial time.

With the optimization question answered, Vašek turned his attention to possible characterizations of perfect graphs. As David has mentioned, one of his approaches involved obtaining characterizations via vertex orderings. This work is discussed in Ryan Hayward's paper in this volume [61]. We will focus on a different approach. We introduce it with a quote from a tribute to Berge written by Vašek [35]:

There are theorems that elucidate the structure of objects in some class $\mathbf{C}$ by showing that every object in $\mathbf{C}$ has either a prescribed and relatively transparent

\footnotetext{
4 To prove this statement it is enough to prove it for integer valued $w$ (since we can approximate reals by rational and renormalize rationals to integers). For any such $w$, he considered an associated graph $G_{w}$ whose vertex set is the union of stable sets $S_{1}, \ldots S_{n}$ where $\left|S_{i}\right|=w_{i}$ and $u \in S_{i}$ is joined to $v \in S_{j}$ precisely if $v_{i} v_{j}$ is an edge of $G$. It is not hard to see that the weight of a maximum weight stable set $x^{*}$ of $G$ is the same as the size of the largest stable set of $G_{w}$. Lovász's result easily implies that $G_{w}$ is perfect, and hence has a covering using $\alpha\left(G_{w}\right)$ cliques. Each of these cliques corresponds in an obvious way to a clique of $G$. So we obtain a set of $w x^{*}$ cliques of $G$ such that each $v_{i}$ is contained in $w_{i}$ of these cliques. The corresponding inequalities of (1) now show that $w x^{*}$ maximizes $w x$ over this polytope.
} 
structure or one of a number of prescribed structural faults along which it can be decomposed.

Vašek decided to apply this paradigm to Berge Graphs, i.e. graphs $G$ such that neither $G$ nor its complement contains an induced odd cycle with at least five vertices. He hoped to thereby both prove the strong perfect graph conjecture and develop a polynomial time recognition algorithm for the class of perfect graphs (see [32] where he first suggested this approach).

With Vašek leading the way, we went to work. I started my M.Sc. in January of 1983 and immediately began to attend a weekly seminar on perfect graphs. Other students of Vašek who attended were Chính Hoàng, Ryan Hayward, and Stefan Olariu. David Avis, Sue Whitesides, and Jean-Marie Bourjolly put in occasional appearances. The atmosphere was electric. The seminars were scheduled for three hours but could go on for much longer. If we started to play backgammon they could last all night.

Initially, Vašek simply gave us a long list of problems and asked us to try and solve them. The seminar was devoted to the presentation of solutions and the discussion of approaches. Through solving the problems, we learnt of a number of special types of perfect graphs to which the paradigm had already been successfully applied: triangulated graphs had been decomposed using clique cutsets; comparability graphs had been decomposed using homogeneous sets.

Once we were sufficiently bloodied, the Master began to educate us in earnest. Fonlupt and Uhry had recently decomposed the class of graphs in which every odd cycle of length five had at least two chords (these graphs were called Meyniel, as it was Meyniel who proved they were perfect), using the so-called amalgam decomposition. Sensei prepared a crystal clear set of notes explaining their complicated decomposition procedure and led us through them.

It was in the spring of 1983 that Vašek introduced two important notions: star cutsets and skew partitions. A star cutset is a cutset containing a vertex $v$ which sees all the other vertices of the cutset. A skew partition is a partition of $V(G)$ into two sets $A$ and $B$ such that $A$ induces a disconnected subgraph of $G$ and $B$ induces a disconnected subgraph of $\bar{G}$. We note that if $(A, B)$ is a skew partition of $G$ then $(B, A)$ is a skew partition of $\bar{G}$. We also note that if $G$ has a star cutset $B$ with at least two vertices then $(V-B, B)$ is a skew partition of $G$.

In a seminal paper [34], Vašek proved that no minimal imperfect graph contains a star cutset. He also observed that this result implied that minimal imperfect graphs had no homogenous sets, had no clique cutsets, and did not permit amalgam decompositions. Thus star cutsets could be used to prove the perfection of almost all the special classes of Berge graphs which had been shown to be perfect. Spurred on by Vašek, Ryan Hayward [60] proved that weakly triangulated graphs ${ }^{5}$ are perfect using the existence of star cutsets in these graphs. These last two results suggested that star cutsets were a powerful tool. Vašek thought that skew partitions might be even more important because of their self-complementarity, which mirrored this property of perfect graphs. In [34], he conjectured that no minimal perfect graph permits a skew partition.

${ }^{5} \mathrm{G}$ is weakly triangulated if neither $G$ nor $\bar{G}$ contains an induced cycle of length at least five. 
Motivated by self-complementarity, he turned his attention to bull-free Berge graphs. A bull is the graph obtained from a triangle by adding two pendant vertices attached to distinct vertices of the triangle; it is self-complementary. Vašek, working with Najiba Sbihi, proved that bull-free Berge Graphs are perfect [45]. In order to do so, they needed to introduce a new decomposition, the homogenous pair ${ }^{6}$. They showed that no minimal imperfect graph contains a homogeneous pair. Using this decomposition and star cutsets, they managed to show that every bull-free Berge graph is perfect.

Vašek's intuition as to the decompositions needed to prove the strong perfect graph conjecture were spot on. Indeed, Chudnovsky, Robertson, Seymour, and Thomas recently proved that every Berge graph either:

(a) is in one of five basic classes of perfect graphs (line graphs of bipartite graphs, their complements, bipartite graphs, their complements, or double split graphs), or

(b) permits one of three partitions (a proper 2-join, a homogeneous pair, or a special type of skew partition which they call balanced $d^{7}$ ).

Of the three decompositions they used, two were defined by Vašek and the third was also one on which he focused the perfect graph community's attention. Indeed, Vašek and I co-organized a conference on perfect graphs in Princeton in 1993. The only talks were by Conforti and Cornuejols who had recently decomposed balanced graphs using 2-joins and some other star-cutset like decompositions. We thought that these ideas could be the last pieces needed to solve the perfect graph puzzle. This was indeed the case. Thus Vašek's vision as always was excellent, and the work carried out in those heady days in the mid 1980s played an important role in the resolution of the strong perfect graph conjecture.

Of course, Vašek was not satisfied with just studying perfect graphs during this period (see [44]). He would not be tied down in this way [36]. He went to the World Backgammon Championship in 1984, organized the first mathematical workshop at McGill's Bellairs Research Institute, made friends with J.P. Donleavy and Panther, took his cubs to a conference in Hakone organized by Jin Akiyama, and wrote a book which beginners could use to learn the first two hundred kanji.

He also maintained two interests David has already mentioned: trying to find large classes of problems which require exponential time to solve when attacked using a standard approach, and obtaining solutions to integer programs from solutions to their fractional relaxations. In [46], he and Szemerédi showed that for every $k \geq 3$ and $c \geq 2^{k}$, almost every satisfiability instance of $c n$ clauses is unsatisfiable but any resolution-based proof of its unsatisfiability requires exponential time. In [28], he showed that the chromatic number of any graph is $O(\log n)$ times its fractional

\footnotetext{
${ }^{6}$ A homogenous pair consists of two disjoint sets of vertices $S_{1}$ and $S_{2}$ such that every vertex outside of $S_{1} \cup S_{2}$ is adjacent to all of $S_{1}$ or none of $S_{1}$ and is adjacent to all of $S_{2}$ or none of $S_{2}$. Furthermore, we insist that $3 \leq\left|S_{1} \cup S_{2}\right| \leq|V|-2$.

7 The first balanced skew cutsets which were shown not to occur in perfect graphs were the $T$-cutsets. This was done by Chinh Hoang while he was a student of Vašek [62]. For more on the history of this type of skew cutset and the role Vašek played see [71].
} 
chromatic number ${ }^{8}$. Actually, he proved something stronger. He showed that a simple greedy heuristic could find a solution to any weighted set cover ${ }^{9}$ problem whose size was at most $\log n$ times the cost of an optimal fractional solution.

I graduated in 1986; it was a third of a lifetime ago and Vašek was 40. David had been like a brother to Vašek, I felt more like a son. This was not because Vašek had put distance between us. It was against Vašek's religion to pretend that his position and academic credentials somehow put him on a different plane from his students or anyone else. Even the standard roping in of graduate students for joe jobs in return for all the time you invested in them was anathema to him. There was an immediateness, integrity and essential humanity in Vašek's interactions, which few if any other supervisors would have matched. And we spent a lot of time playing as equals: bedtime stories at the Ritz, randomly chosen shops in Tokyo, the Salon de Livres in Paris and Chez Paris, Nena Hagen in both English and German, and Blondie's immortal

I'm in the phone booth, it's the one across the hall. If you don't answer then I'll just ring it off the wall.

Despite this closeness, Vašek was above all my mentor. Indeed I had been floundering and unmotivated as an undergraduate. My decision to go to graduate school and become an academic was inspired solely by my exposure to his brilliant lectures and charming wit when I took a course from him in my last year.

His mentoring continued throughout graduate school. We had countless meals at the Coffee Mill on Mountain Street. After acknowledging the waitress with their standard exchange ${ }^{10}$, lighting a cigarette, and starting to eat, he would begin to explain a new theorem to me. Possibly one of his results. Possibly a breakthrough obtained by another researcher. These recitals never failed to fascinate me and I spent as much time with Vašek as he allowed.

I hope that the reader who thinks I have spent too much time discussing my relationship with Vašek will forgive me. I have done so because I do not believe my experience was unique. Vašek brought magic into my life, from what colleagues have told me, others feel touched by this magic even if they have just listened to one of his lectures or chatted with him for $15 \mathrm{~min}$.

In short then, during my years as a student, Vašek was a pussycat. I often find myself, when faced with a decision as to what to do as a supervisor asking: what would the kitty do? I do not always live up to his example but I am a better man for trying.

\footnotetext{
8 A fractional $\alpha$-colouring is an assignment of non-negative weights to the stable sets of $G$ so that the sum of the weights of the stable sets containing each vertex is one. The fractional chromatic number is the minimum $\alpha$ for which $G$ has a fractional $\alpha$-colouring.

9 In a set cover problem we are given some subsets of a ground set and need to find the minimum number of subsets whose union contains the ground set. In graph colouring, the ground set is the vertex set and the subsets are the stable sets.

${ }^{10}$ Greetings Comrade, formerly Hello.
} 


\section{Travelling Salesman-Notes by WC}

In a typically modest fashion, Vašek [4] introduced our computational work on solving instances of the travelling salesman problem.

Dantzig, Fulkerson, and Johnson showed a way to solve large instances of the TSP; all that came afterward is just icing on the cake. The purpose of the present paper is to describe some of the icing we have added on top of the previous layers.

This short remark was the subject of much discussion, leading to pictures of cakes in lectures [74] and writings [1, 2, 78].

The TSP asks for a minimum cost Hamiltonian circuit, or tour, in a graph $G=(V, E)$ with edge costs; the original 1954 paper of Dantzig et al. [49] presents the solution of a 42-city instance of the problem via a sequence of LP relaxations. Dantzig et al. record a tour as a $0-1$ vector $x=\left(x_{e}: e \in E\right)$, where $x_{e}=1$ if and only if $e$ is in the tour. An LP relaxation for the TSP consists of a family of linear inequalities satisfied by all tour vectors. A modest-sized starting point consists of bounds $0 \leq x_{e} \leq 1$, for each edge $e$, and equations

$$
\sum\left(x_{e}: e \text { meets vertex } v\right)=1,
$$

for each vertex $v$. Such an LP can be easily solved with the simplex algorithm, and a dual solution provides a lower bound on the cost of any tour. To improve this bound, Dantzig et al. locate additional inequalities that are valid for all tours but violated by the LP optimal solution $x^{*}$. A selection of these inequalities, called cutting planes, or just cuts, are added to the relaxation and the process is repeated. The primary supply of cuts in [49] are the subtour constraints

$$
\sum\left(x_{e}: e \text { has exactly one end in } S\right) \geq 2
$$

for each proper subset $S \subset V$. A collection of these cuts were found by hand in their work. Together with two ad hoc inequalities, the subtour cuts formed a relaxation that established the optimal value of a tour through their 42 cities.

Vašek was of course in a perfect position to focus attention on the ground breaking work of Dantzig et al. It was Vašek [19] himself who introduced the concept of comb inequalities that brought the cutting-plane approach to new heights, following Gomory's [55] call to action and Hong's [63] branch-and-cut computer code. Vašek's theme was taken up by Martin Grötschel and Manfred Padberg [59], who championed the use of combs in the TSP in the late 1970s and 1980s.

In Grötschel and Padberg's work a comb consists of subsets $T_{0}, T_{1}, \ldots, T_{s}$ of $V$, such that $s$ is odd and at least 3 , the sets $\left(T_{j}: j=1, \ldots, s\right)$ are pairwise disjoint, and for each $j \geq 1$ the set $T_{j}$ contains at least one vertex in $T_{0}$ and at least one vertex not in $T_{0}$. Vašek called $T_{0}$ the handle and $\left(T_{j}: j=1, \ldots, s\right)$ the teeth of the comb; in his version each tooth must intersect the handle in exactly one vertex. Every TSP tour satisfies the comb inequality

$$
\sum_{j=0}^{s} \sum\left(x_{e}: e \text { has exactly one end in } T_{j}\right) \geq 3 s+1 \text {. }
$$


The inclusion of combs as a source of cutting planes is a powerful extension of the subtour cuts, as can be seen in the success of the Grötschel-Padberg-led work $[48,56,57,70]$. Along with these computations, combs have also been the focus of many theoretical studies. An important open question here is to determine the complexity of the separation problem for combs, that is, given an LP vector $x^{*}$, find a violated comb inequality if one exists; no polynomial-time algorithm is known for the problem and it is also not known to be $N P$-hard.

After the publication of Vašek's comb paper in 1974, he remained interested in the TSP, mainly through research on Hamiltonian graphs. In was in Oberwolfach, January 1987, where we began our TSP work together. At an evening problem session, Martin Grötschel discussed several questions regarding the Chvátal rank of the subtour relaxation of the TSP. The subtour relaxation consists of all subtour inequalities, together with the Eq. (1), for all vertices, and the bounds $0 \leq x_{e} \leq 1$, for all edges. The main question posed by Grötschel was to show that the Chvátal rank of the relaxation cannot be bounded above by any constant as the number of vertices increases. It was known that the rank was at least two, but no further lower bounds were available.

After a brisk walk down to the village pub, Vašek proposed that we jump on the problem, feeling that a couple of glasses of beer, several kirschwassers, and a study of non-Hamiltonian graphs would be a good place to start. As usual, Vašek was right on the money; a class of graphs from his flip-flop paper [21] was just what the doctor ordered. When a break was called for we joined in a few songs with the locals at the pub, who quickly pronounced Vašek as the most likeable mathematician ever to set foot in their fair valley. We managed to come away with enough notes to settle Grötschel's problem, showing that the Chvátal rank grew at least linearly with the number of vertices.

The subtour relaxation result was the starting point for a lengthy study of lower bounds on the rank of other polyhedra, carried out by Vašek, Mark Hartmann, and myself [37]. As this work drew to a close, it seemed logical to attempt to turn the ideas into an attack on solving instances of the TSP. Inspiration here came from a second session from the 1987 Oberwolfach meeting, where Manfred Padberg presented fresh work with Giovanni Rinaldi on the computation of an optimal tour for a 2,392-city instance. We thought we had enough tricks up our sleeves to give the area another push, and decided on February 27, 1988, that it was time to give it a go.

The next day we purchased a desktop PC from a vendor in lower Manhattan. Curiously, when the main technician at the shop learned that we were mathematicians he warned "You guys aren't going to try to solve that travelling salesman problem, are ya?" But that is just what we had in mind. A few days later we contacted Dave Applegate, a star PhD student working with Ravi Kannan at Carnegie Mellon, and the project was up and running. Initially we attempted various combinatorial techniques for obtaining lower bounds, but we gradually drifted back towards traditional LP-based methods. This period came to a head in a crash coding session over two days in April 1990 in Montreal, producing a simplex algorithm specially tailored for the TSP. Although this worked fairly well, the code could not beat Bob Bixby's newly released general simplex implementation CPLEX 1.0. 
Later in April 1990 we arrived at Rice University for a TSP Workshop organized by Bob Bixby at the Center for Research in Parallel Computing. Our work was a bit of a mystery, but it was known that we had been studying the TSP for the past two years. When our session came around, Vašek began his lecture with a line by line history of the largest solved instances: Dantzig-Fulkerson-Johnson 42 cities, Held-Karp 57 cities, . . , Padberg-Rinaldi 2,392 cities. Keeping the bottom portion of his slide covered, he noted the point in 1988 where we "got into the game." With the crowd on its heels, he slowly revealed the final line: Applegate-Chvátal-Cook 17 cities. This was an exaggeration of the state of our project, but it was true that we had more ideas than computational results at that point.

In the aftermath of the meeting, Vašek kept reminding us that you need to get up pretty early in the morning if you are going to beat the simplex algorithm. We thus made a wise move and convinced Bob Bixby to join our project. With Bob churning out ever faster versions of the LP solver, we focused our attention on the generation of cutting planes to keep the solver busy.

In this work, Vašek viewed the search for cuts as a two-step process. First, by fair means or foul, he would locate a flaw in our current LP solution $x^{*}$, that is, a property of $x^{*}$ that could not be possessed by a convex combination of tours. Second, following the theme of his cutting-plane work in the 1970s, he would find a way to expose this flaw via a linear inequality. This is the method employed in a heuristic separation algorithm for combs based on the consecutive ones property ([5], Chap. 8). In this case, the flaw is specified by a family $\mathcal{F}$ of subsets of vertices such that each member $S \in \mathcal{F}$ satisfies

$$
\sum\left(x_{e}^{*}: e \text { has exactly one end in } S\right)=2,
$$

no member of $S$ contains vertex $v_{0}$, and $\mathcal{F}$ does not have the consecutive ones property. To spot such a flaw, we can employ the linear-time algorithm of Booth and Lueker [10] for testing the consecutive ones property. An immediate cutting plane exposing $\mathcal{F}$ is

$$
\sum_{S \in \mathcal{F}} \sum\left(x_{e}: e \text { has exactly one end in } S\right) \geq 2|\mathcal{F}|+2,
$$

but this can be weak in the sense that it may be the sum of smaller valid inequalities. A nice twist, however, follows from an old theorem of Tucker [76], implying that a minimal consecutive-ones flaw $\mathcal{F}$ is in fact a three-toothed comb, yielding a method to spot these much sought-after cuts.

Ideas such as the consecutive-ones heuristic were usually the fruit of long nights in cafes, staring at drawings of $x^{*}$ vectors for potential flaws. As the TSP project continued, Vašek became convinced that the entire two-step discovery process could be automated, at least to some degree. Given the usually dim cafe lighting, the search for flaws was often limited to local regions of $x^{*}$, with the remainder of the vector considered as one super-vertex that could be visited any even number of times. This shrinking of the vector can be viewed as linear mapping $\phi$ that takes an LP solution into a space of relatively small dimension. If the region under examination is small enough, then a computer code should be able to determine if $\phi\left(x^{*}\right)$ can be 
written as a convex combination of mapped tours, and, if not, produce a cutting plane $a^{T} \bar{x} \leq b$ in the mapped space. By substitution, any mapped inequality gives a cutting plane $a^{T} \phi(x) \leq b$ for $x^{*}$. Vašek worked out examples by hand showing that this general framework could produce strong TSP cuts, and the method grew into the local cuts procedure ([5], Chap. 11) that is incorporated into our Concorde code. This in turn has led to the use of local cuts in other problem areas, such as Steiner-tree computations [3] and mixed-integer programming [52].

Vašek also pursued the idea of turning validity proofs of inequalities into algorithms for producing cutting planes. The technique is to take apart the proof and build structures that allow each step to be carried out constructively. An example here is the necklace heuristic for combs ([5], Chap. 8), modelled after the algebraic proof of comb inequalities as a Chvátal-Gomory cut. The basic object in this case is a domino, consisting of a pair $(A, B)$ of subsets of vertices such that $A \cap B=\emptyset$ and $A \cup B \neq V$. Dominoes are candidates for teeth in a comb, where $A$ is the set of vertices in the handle and $B$ is the set of vertices not in the handle. The heuristic works with certain large families of dominoes, selecting odd sets for the teeth by solving linear systems over $G F(2)$. The linear systems produce multipliers needed in a Chvátal-Gomory derivation of a comb from subtour constraints. This method has been pushed further in work by Fleischer and Tardos [53] and Letchford [67], and it has served as one of the starting points for the study of mod-2 cuts in other problem areas $[12,13,73]$.

All of this cutting-plane work and more was carried out with the concrete aim of solving large instances of the TSP. Starting with the solution of a 3,038-city example in 1992, improved versions of the Concorde code gradually worked through the entire TSPLIB [72] challenge set. The last instance, consisting of 85,900 cities from a VLSI application, fell in 2006. This success is a triumph of Vašek's ideas, but his legacy goes way beyond these computations. The mathematical elegance Vašek brought to the TSP will guide computational work long after new TSP records have

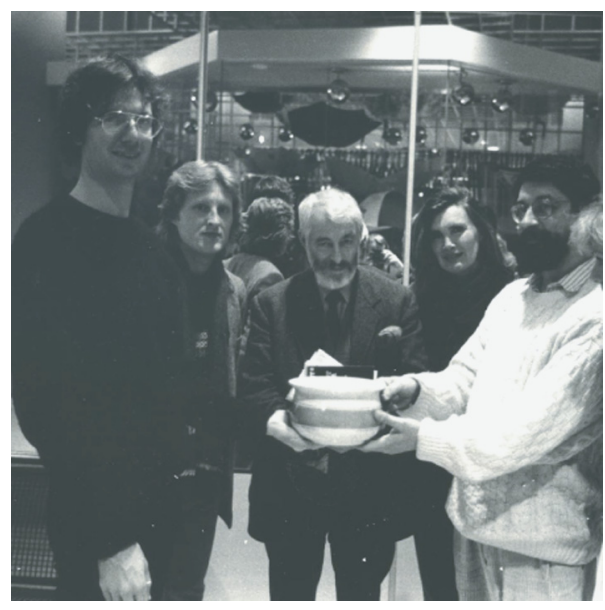

Fig. 12. Donleavy got his pot to piss in, taken by Adrian 


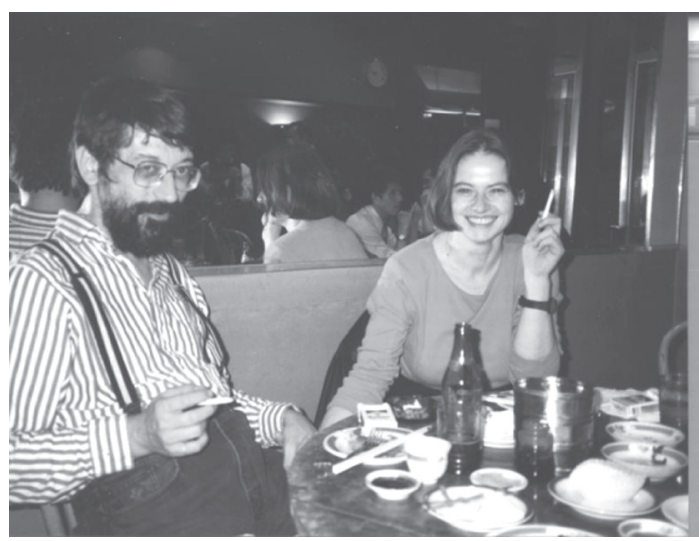

Fig. 13. With Marketa

come and gone. It is difficult to predict where TSP research will head in the coming decades, but it is certain that progress will continue if the community follows Vašek's lead and bashes on regardless (Figs. 12, 13).

Acknowledgements. We would like to thank Ryan Hayward for sharing his recollections, and for a careful reading of the manuscript.

\section{References}

1. Aardal, K.: 7397-city traveling salesman instance solved-another layer of icing on the cake. Optima 45 (1995)

2. Aardal, K.: TSP Cake. Optima 61, 20-21 (1999)

3. Althaus, E., Polzin, T., Daneshmand, S. V.: Improving linear programming approaches for the Steiner tree problem. Lect. Notes Comput. Sci. 2647, 1-14 (2003)

4. Applegate, D., Bixby, R., Chvátal, V., Cook, W.: Finding cuts in the TSP (a preliminary report). DIMACS Technical Report 95-05. DIMACS, Rutgers University, New Brunswick, NJ, USA, 1995

5. Applegate, D. L., Bixby, R. E., Chvátal, V., Cook, W. J.: The Traveling Salesman Problem: A Computational Study. Princeton University Press, Princeton (2006)

6. Avis, D., Chvátal, V.: Notes on Bland's pivoting rule. Math. Program. Study 8, 24-34 (1978)

7. Bauer, D., Broersma, H. J., Veldman, H. J.: Not every 2-tough graph is Hamiltonian. Discrete Appl. Math. 99, 1-3 (2000)

8. Berge, C.: Some classes of perfect graphs. In: Six papers on Graph Theory, pp. 1-21. Indian Stastical Institute, Calcutta (1963)

9. Bloom, D. M., Benkoski, S., Chvátal, V., Forsey, H., Demir, H.: Problems and Solutions: Elementary Problems: E2298, E2307-E2312. Am. Math. Mon. 78, 792-793 (1971)

10. Booth, K.S., Lueker, G. S.: Testing for the consecutive ones property, interval graphs, and graph planarity using PQ-tree algorithms. J. Comput. Syst. Sci. 13, 335-379 (1976)

11. Bondy, J. A., Chvátal, V.: A method in graph theory. Discrete Math. 15, 111-135 (1976)

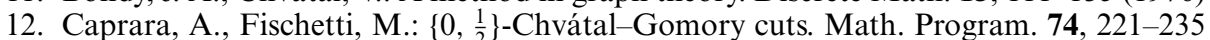
(1996)

13. Caprara, A., Fischetti, M., Letchford, A. N.: On the separation of maximally violated mod- $k$ cuts. Math. Program. 87, 37-56 (2000) 
14. Chvátal, V.: On finite and countable rigid graphs and tournaments. Comment. Math. Univ. Carol. 6, 429-438 (1965)

15. Chvátal, V.: The smallest triangle-free 4-chromatic 4-regular graph. J. Comb. Theory 9 , 93-94 (1970)

16. Chvátal, V.: Hypergraphs and Ramseyian theorems. Proc. Am. Math. Soc. 27, 434-440 (1971)

17. Chvátal, V.: On Hamilton's ideals. J. Comb. Theory B 12, 163-168 (1972)

18. Chvátal, V.: Monochromatic paths in edge-colored graphs. J. Comb. Theory B 13, 69-70 (1972)

19. Chvátal, V.: Edmonds polytopes and weakly Hamiltonian graphs. Math. Program. 5, 29-40 (1973)

20. Chvátal, V.: Edmonds polytopes and a hierarchy of combinatorial problems. Discrete Math. 4, 305-337 (1973)

21. Chvátal, V.: Flip-flops in hypoHamiltonian graphs. Can. Math. Bull. 16, 33-41 (1973)

22. Chvátal, V.: Tough graphs and Hamiltonian circuits. Discrete Math. 5, 215-218 (1973)

23. Chvátal V.: Intersecting families of edges in hypergraphs having the hereditary property. Hypergraph Seminar Proceedings of the First Working Seminar, Ohio State University, Columbus, Ohio. Lect. Notes Math. 411, 61-68 (1974)

24. Chvátal, V.: On certain polytopes associated with graphs. J. Comb. Theory B 18, 138-154 (1975)

25. Chvátal, V.: A combinatorial theorem in plane geometry. J. Comb. Theory B 18, 39-41 (1975)

26. Chvátal, V.: Some linear programming aspects of combinatorics. Congr. Numer. 13, 2-30 (1975)

27. Chvátal, V.: Determining the stability number of a graph. SIAM J. Comput. 6, 643-662 (1977)

28. Chvátal V.: A greedy heuristic for the set-covering problem. Math. Oper. Res. 4, 233-235 (1979)

29. Chvátal V.: Hard knapsack problems. Oper. Res. 28, 1402-1411 (1980)

30. Chvátal V.: Linear Programming. W.H. Freeman, New York (1983)

31. Chvátal, V.: Cutting-plane proofs and the stability number of a graph. Report 84326 , University of Bonn, pp. 10 (1984)

32. Chvátal, V.: Notes on perfect graphs. In: Pulleyblank WR (ed.) Progress in Combinatorial Optimization, pp. 107-115. Academic, New York (1984)

33. Chvátal, V.: In praise of Claude Berge. Discrete Math. 165/166, 3-9 (1997)

34. Chvátal, V.: Star-cutsets and perfect graphs. J. Comb. Theory B 39, 189-199 (1985)

35. Chvátal, V.: Claude Berge 5.6.1926-30.6.2002. Graphs Comb. 19, 1-6 (2003)

36. Chvátal, V., Cook, W.: The discipline number of a graph. Discrete Math. 86, 191-198 (1990)

37. Chvátal, V., Cook, W., Hartmann, M.: On cutting-plane proofs in combinatorial optimization. Linear Algebra Appl. 114/115, 455-499 (1989)

38. Chvátal, V., Erdős P.: A note on Hamiltonian circuits. Discrete Math. 2, 111-113 (1972)

39. Chvátal, V., Harary, F.: Generalized Ramsey theory for graphs. I. Diagonal numbers. Periodica Mathematica Hungarica. J. János Bolyai Math. Soc. 3, 115-124 (1973)

40. Chvátal, V., Harary, F.: Generalized Ramsey theory for graphs. II. Small diagonal numbers. Proc. Am. Math. Soc. 32, 389-394 (1972)

41. Chvátal, V., Harary, F.: Generalized Ramsey theory for graphs. III. Small off-diagonal numbers. Pacific J. Math. 41, 335-345 (1972)

42. Chvátal, V., Klarner, D. A., Knuth, D. E.: Selected Combinatorial Research Problems. Computer Science Department, Stanford University, CS-TR-72-292, 1972

43. Chvátal, V., Lovász, L.: Every directed graph has a semi-kernel. Hypergraph Seminar Proceedings of the First Working Seminar, Ohio State University, Columbus, Ohio. Lect. Notes Math. 411, 175 (1974) 
44. Chvátal, V., Reed, B.: Mick gets some (the odds are on his side). In: Proceedings of the 33rd Annual Symposium on Foundations of Computer Science, pp. 620-627. IEEE Computer Society Press, Washington (1992)

45. Chvátal, V., Sbihi, N.: Bull-free Berge graphs are perfect. Graphs Combin. 3, 127-139 (1987)

46. Chvátal, V., Szemerédi, E.: Many hard examples for resolution. J. Assoc. Comput. Mach. 35, 759-768 (1988)

47. Cook, S. A.: The complexity of theorem-proving procedures. 3rd ACM Symposium on Theory of Computing, pp. 151-158, 1970

48. Crowder, H., Padberg, M. W.: Solving large-scale symmetric travelling salesman problems to optimality. Manage. Sci. 26, 495-509 (1980)

49. Dantzig, G., Fulkerson, R., Johnson, S.: Solution of a large-scale traveling-salesman problem. Oper. Res. 2, 393-410 (1954)

50. Deza, M., Laurent, M.: Geometry of Cuts and Metrics. Springer, Berlin (1997)

51. Edmonds, J.: Maximum matching and a polyhedron with 0,1-vertices. J. Res. Natl. Bur. Stand. 69B, 125-130 (1965)

52. Espinoza, D. G.: On Linear programming, Integer Programming and Cutting Planes Ph.D. Thesis. School of Industrial and Systems Engineering, Georgia Institute of Technology, Atlanta, Georgia, USA, 2006

53. Fleischer, L., Tardos, É.: Separating maximally violated comb inequalities in planar graphs. Math. Oper. Res. 24, 130-148 (1999)

54. Gomory, R. E.: An algorithm for integer solutions to linear programs. Princeton IBM Math Report, 1958

55. Gomory, R. E.: The traveling salesman problem. In: Proceedings of the IBM Scientific Computing Symposium on Combinatorial Problems, pp. 93-121. IBM, White Plains (1966)

56. Grötschel, M.: On the symmetric travelling salesman problem: solution of a 120-city problem. Math. Program. Study 12, 61-77 (1980)

57. Grötschel, M., Holland, O.: Solution of large-scale symmetric travelling salesman problems. Math. Program. 51, 141-202 (1991)

58. Grötschel, M., Lovász, L., Schrijver, A.: The ellipsoid method and its consequences in combinatorial optimization. Combinatorica 1, 189-197 (1981)

59. Grötschel, M., Padberg, M. W.: On the symmetric traveling salesman problem. I: Inequalities. Math. Program. 16, 265-280 (1979)

60. Hayward, R.: Weakly triangulated graphs. J. Comb. Theory B 39, 200-208 (1985)

61. Hayward, R.: The story of perfectly orderable graphs. Graphs Comb. (this volume)

62. Hoàng, C.: Some properties of minimal imperfect graphs. Discrete Math. 160, 165-175 (1996)

63. Hong, S.: A Linear Programming Approach for the Traveling Salesman Problem. Ph.D. Thesis. The Johns Hopkins University, Baltimore, MD, USA, 1972

64. Karp, R. M.: Reducibility among combinatorial problems. In: Miller, R. E., et al. (eds.) Complexity of Computer Computations. (1972)

65. Khachian, L. G.: A polynomial time algorithm in linear programming. Doklady Akademiiia Nauk SSSR 244, 1093-1096 (1980)

66. Lawler, E. L., Lenstra, J. K., Rinnooy Kan, A. H. G., Shmoys, D. B. (eds.): The Traveling Salesman Problem. Wiley, New York (1985)

67. Letchford, A. N.: Separating a superclass of comb inequalities in planar graphs. Math. Oper. Res. 25, 443-454 (2000)

68. Lovász, L.: Normal hypergraphs and the perfect graph conjecture. Discrete Math. 2 , 253-267 (1972)

69. Lovász, L.: A characterization of perfect graphs. J. Comb. Theory B 13, 95-98 (1972)

70. Padberg, M., Rinaldi, G.: A branch-and-cut algorithm for the resolution of large-scale symmetric traveling salesman problems. SIAM Rev. 33, 60-100 (1991)

71. Reed, B.: Skew partitions in perfect graphs. Discrete Appl. Math. (in press) 
72. Reinelt, G.: TSPLIB - a traveling salesman problem library. ORSA J. Comput. 3, 376384 (1991)

73. Reinelt, G., Wenger, K. M.: Maximally violated mod- $p$ cuts for the capacitated vehiclerouting problem. INFORMS J. Comput. 18, 466-479 (2006)

74. Padberg, M.: Plenary Lecture, XVI International Symposium on Mathematical Programming, Lausanne, August (1997)

75. Shannon, C. E.: The zero error capacity of a noisy channel. Institute of Radio Engineers. Trans. Inf. Theory IT-2, 8-19 (1956)

76. Tucker, A.: A structure theorem for the consecutive 1's property. J. Comb. Theory B 12, 153-162 (1972)

77. Underground, P.: Graphs with Hamiltonian squares. Discrete Math. 21, 323 (1978)

78. Yamamoto, Y., Kubo, M.: Invitation to the Traveling Salesman Problem. Asakura, Tokyo, 1997 (in Japanese)

Received: January, 2007

Revised version received:

Query:

1) Please provide page numbers for Ref. [1].

2) Please update Ref. [71].

3) The Ref. $[9,27,66]$ have not been cited in the text. Please provide text citations for the above references, or delete the same from reference list.

4) Please check figure citations and placements of Figures 1-13. 